\title{
Helcococcus ovis in a patient with an artificial eye: a case report and literature review
}

\author{
Liyan Mao, Zhongju Chen, Yanfang Lu, Jing Yu, Yu Zhou, Qun Lin, Ying Luo and Ziyong Sun*
}

\begin{abstract}
Background: Helcococcus ovis, belonging to the genus of Helcococcu in Peptostreptococcaceae, is one kind of facultative anaerobic and gram-positive cocci, which was first isolated from a mixed infection in sheep in 1999. To our knowledge, it's known as an invasive pathogen in animals, and never been reported as a human pathogen in published literature. The aims of this work are to describe the first report of $H$. ovis which was recovered from the artificial eye of human case and perform a literature review.

Case presentation: A 26 year-old man reporting pyogenic infection with an artificial eye attended ophthalmic ward in Tongji hospital. After physical examination, clinical and laboratory investigations, the diagnosis of eye infection caused by Helcococcus ovis and Staphylococcus aureus was established. Receiving a medico-surgical approach, the patient was successfully treated. The treatment consisted in intravenous cefotaxime and ornidazole, levofloxacin eye drops during two weeks and removing of right artificial eye with debridement.

Conclusions: We describe here the first known case of $\mathrm{H}$. ovis which was recovered from human artificial eye. This report different from previous data found in the literature emphasizes the invasive potential of this bacterial species as a pathogen in human. Prospectively, the application of next generation sequencing tools would contribute to a more accurate classification of clinical strains.
\end{abstract}

Keywords: Eye infection, Artificial eye, Helcococcus ovis, 165 rRNA gene sequencing, Antimicrobial susceptibility

\section{Background}

Helcococcus, a facultatively anaerobic, catalase-negative, Gram-positive cocci, was first described in 1993 by Collins and colleagues [1]. This genus composes of five species, namely Helcococcus kunzii (first reported in 1993 [1]), Helcococcus ovis (first reported in 1999 [2]), Helcococcus pyogenica (first reported in 2004 [3]), Helcococcus sueciensis (first reported in 2004 [4]) and Helcococcus seattlensis (first reported in 2014 [5]). All species, with the exception of H. ovis, have been isolated from human specimens [3-8], and $H$. kunzii is the most common pathogen [7-10]. H. ovis was first isolated from a mixed infection in sheep in 1999 and was subsequently reported in bovine, horses and goats [2], but has never been isolated from human specimens, even as a result of foreign body invasion [11, 12]. In this report, we describe

\footnotetext{
* Correspondence: tjszyong@163.com

Department of Laboratory Medicine, Tongji Hospital, Tongji Medical College,

Huazhong University of Science and Technology, Wuhan 430030, China
}

the first known human case of artificial eye infection, which H. ovis was isolated from the artificial eye.

\section{Case presentation}

A 26 year-old man attended our ophthalmic ward in April 2017 with intermittent bleeding of the right eye, from which there was also strong odor. The patient was a heavy smoker but had no other underlying conditions. He had no history of drug-use. From his medical history it was noted that the patient had undergone a right ophthalmectomy 24 years previously due to retinoblastoma, and implantation of an artificial right eyeball in 2014 (timeline shown in Additional file 1).

On admission, his pulse rate was between 80 and 100 beats/min. His body temperature and respiratory rate were both normal. Physical examination showed narrow

(C) The Author(s). 2018 Open Access This article is distributed under the terms of the Creative Commons Attribution 4.0 International License (http://creativecommons.org/licenses/by/4.0/), which permits unrestricted use, distribution, and reproduction in any medium, provided you give appropriate credit to the original author(s) and the source, provide a link to the Creative Commons license, and indicate if changes were made. The Creative Commons Public Domain Dedication waiver (http://creativecommons.org/publicdomain/zero/1.0/) applies to the data made available in this article, unless otherwise stated. 
conjunctival sac in right eye and the exposure of ocular prosthesis, which was discharging a yellow-green secretion along with a strong odor. The visual acuity of left eye was 0.3 , and the intraocular pressure was $15 \mathrm{mmHg}$. All other characteristics of the left eye were normal. A auscultation did not show any abnormality in the lungs, and no signs of carotid murmur were found. Interestingly, laboratory investigations did not reveal abnormal inflammatory markers such as leukocytosis or any increase in neutrophils or C-reaction protein. According to clinical and laboratory investigations, infectious endocarditis was not suspected. The patient had no history of other immunosuppressive conditions, except smoking and a retinoblastoma 24 years previously. The patient did not report any direct contact with animals; however, he did work in a clothing factory so would have been contact with wool and cowhide for one month of the year. Three months had elapsed between the patient last coming into contact with wool and cowhide and the appearance of clinical symptoms. Considering the results of these investigations, partial artificial eye infection, especially anaerobic organism infection, was suspected.

Imaging workups were completed, which included chest $\mathrm{x}$-ray, transthoracic echocardiography and eye magnetic

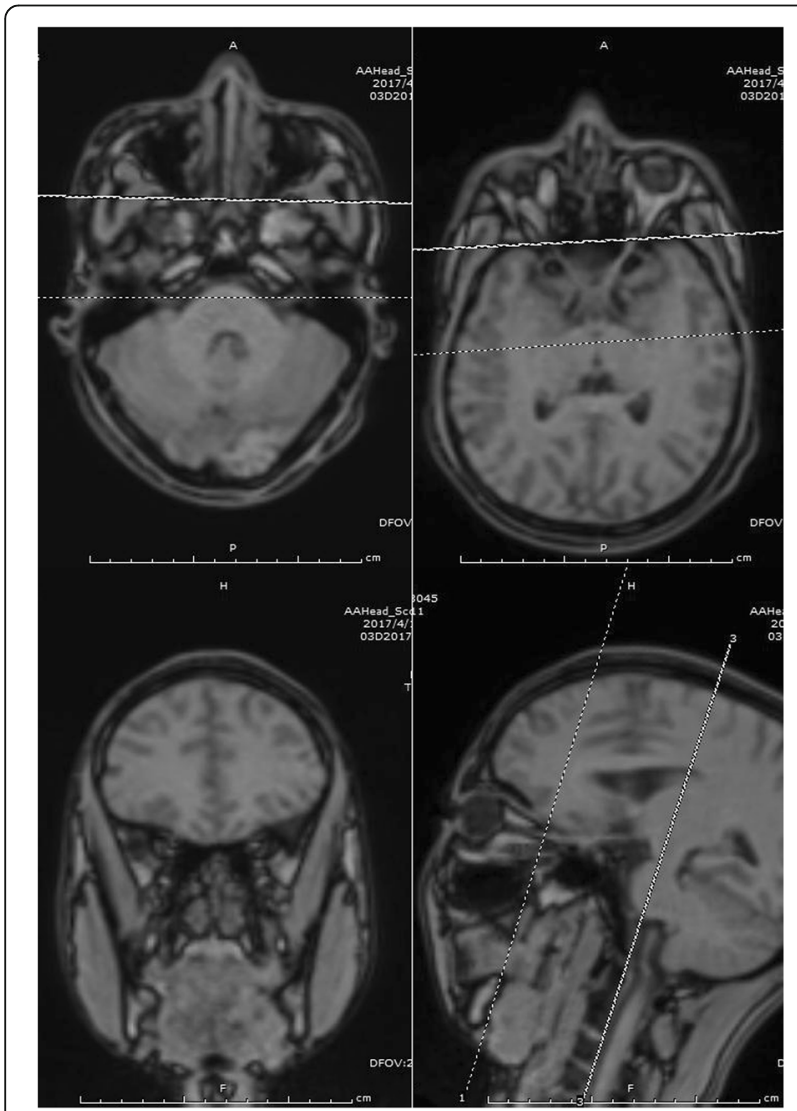

Fig. 1 Eye magnetic resonance imaging of the patient resonance imaging. As shown in Fig. 1, eye magnetic resonance imaging revealed that the tissue surrounding the right eye prosthesis as well as the soft tissue of the lacrimal gland area were swollen, whereas the left eye appeared normal. Inflammatory disease in the right eye was therefore suspected. According to chest $\mathrm{x}$-ray and transthoracic echocardiography, no obvious abnormalities in the lungs or heart were observed.

Before surgery, a few specimens of the right eye secretions were collected to be cultured, but no bacteria were isolated, possibly because most of the secretions had been absorbed by the artifical eye making it yellow-green in appearance. After removal of the right artificial eye with debridement (5 days after admission), both the artificial eye and specimens of the eye secretions were sent for bacterial culture under aerobic and anaerobic conditions. No bacterial growth was detected from the ophthalmic secretions, but cultures were obtained from the artificial eye. Sparse growth of $\beta$-hemolytic cocci and heavy growth of small, non-hemolytic, translucent colonies were observed on Columbia agar plates supplemented with $5 \%$ sheep blood (BioMérieux, Marcy l'Etoile, France) under aerobic conditions after $48 \mathrm{~h}$. And the latter colonies only grow close to the hemolysis zone of the former one. Under anaerobic conditions, only the small, translucent colonies were detected from the artificial eye (as shown in Fig. 2). Of the two colony types, the $\beta$-hemolytic cocci were confirmed as Staphylococcus aureus, whereas the small, translucent colonies stained positive in a Gram stain and occurred singly, in pairs, or in short chains (Fig. 3). Catalase and oxidase reactions of the unknown colonies were negative and phenotypic characterization using the Vitek2 GP system (BioMérieux) was inconclusive. However, Matrix-assisted laser desorption/ionization time of flight (MALDI-TOF) mass spectrometry revealed a match with Helcococcus ovis DSM 21504 T DSM (log score: 1.637) according to the Brucker Maldi-Biotyper database. Identification of this organism was confirmed by $16 \mathrm{~S}$ rRNA gene sequencing. BLAST analysis of the partial $16 \mathrm{~S}$ rRNA gene sequence derived from our isolate (1492 nucleotides, deposited in the GeneBank database under accession number MG188744) showed 98.9\% identity (15 nucleotide differences) with the $16 \mathrm{~S}$ rRNA gene sequence of $\mathrm{H}$. ovis s840-96-2 deposited in the GenBank database under accession number NR027228 by Collins and coworkers [2] in 1999 when this species was first described.

Antimicrobial susceptibility testing (AST) of both strains was performed. The disk diffusion method was carried out and with the exception of penicillin, erythromycin and clindamycin, $S$. aureus isolated from this case was susceptible to all other drugs including cephalosporins and fluoroquinolones. AST for Helcococcus was performed using the CLSI broth microdilution method on Mueller-Hinton II broth (BD Diagnostics, Heidelberg, Germany) supplemented with 


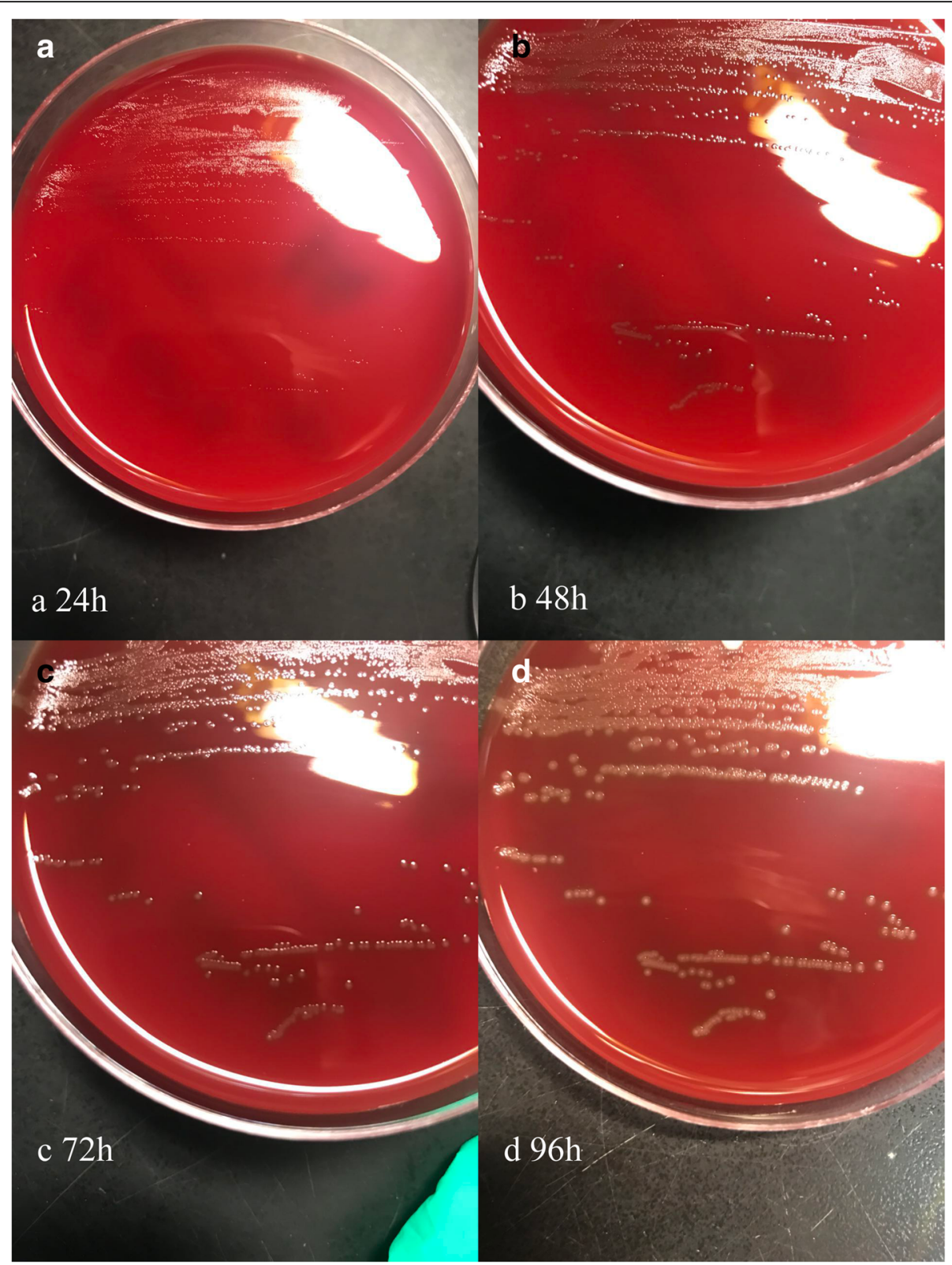

Fig. 2 Colonies of pure Helcococcus ovis on Columbia agar plates supplemented with 5\% sheep blood. a $24 \mathrm{~h}$ after incubation; (b) $48 \mathrm{~h}$ after incubation; (c). $72 \mathrm{~h}$ after incubation; (d) $96 \mathrm{~h}$ after incubation

3\% (vol/vol) lysed horse blood (Oxoid, Wesel, Germany) and $0.001 \%$ (wt/vol) pyridoxal $\mathrm{HCl}$ (Sigma-Aldrich, Munich, Germany) incubated at $37{ }^{\circ} \mathrm{C}$ in $5 \% \mathrm{CO} 2$ for $24 \mathrm{~h}[13,14]$. Streptococcus pneumoniae ATCC 49619 served as a quality control. Then, we changed the method and performed an E-test on blood agar, with S. aureus ATCC 29213 as the quality control (for reference only). The MICs $(\mu \mathrm{g} / \mathrm{L})$ of the drugs for this strain are reported in Table 1. Since no antimicrobial testing guidelines are currently available from the Clinical and Laboratory Standards Institute (CLSI) for Helcococcus, the MICs were determined in reference to the CLSI guidelines for S. aureus [5]. According to the CLSI [5, 15], this strain was susceptible to penicillin, ampicillin, teicoplanin, ceftriaxone, vancomycin, and linezolid.

After admission, the patient received levofloxacin eye drops 4 times per day until being discharged from hospital. After surgery, the patient was initially treated with intra-venous cefotaxime $(2.25 \mathrm{~g} / 250 \mathrm{ml} \mathrm{NaCl}, 1 /$ day $)$ and ornidazole (500 mg/day) for 1 week. Two weeks after admission, the patient recovered and was discharged from hospital. Six months later, the patient returned to the hospital to finish implantation of the 


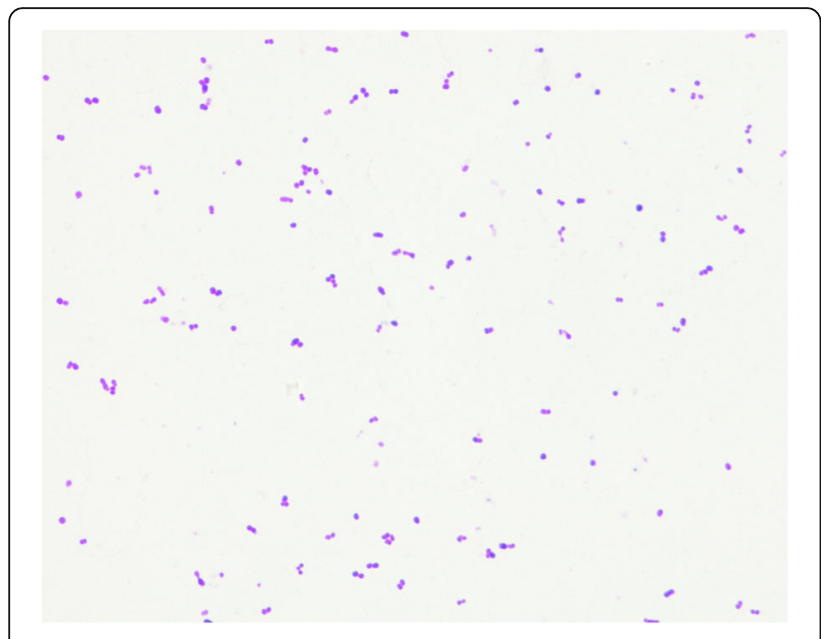

Fig. 3 Gram staining of Helcococcus ovis under oil microscope

artificial right eyeball and no signs of infection were detected. With the patient's consent, we collected samples from the skin around both eyes for aerobic and anaerobic culture, but only normal skin flora were detected, such as coagulase-negative Staphylococcus. After surgery, the patient was in good health and was discharged from the hospital.

\section{Discussion and conclusions}

H. ovis was first described by Collins and colleagues in 1999. Two strains, He.ovis CCUG 374411 and CCUG 39041, were isolated as part of a mixed infection from sheep. The first was from the lungs, liver and spleen at necropsy, whereas the second was from the milk of sheep with subclinical mastitis [2]. Compared with the type strain, Helcococcus kunzii, which was the first member of the Helcococcus genus discovered in 1993 [1], these isolates from sheep were different both in phenotype and genotype, and their sequences were approximately $4 \%$ divergent from that of $H$. kunzii. Therefore, it was suggested that the isolates from sheep be classified as a new species, $H$. ovis.

Briefly, $H$. ovis is a facultatively anaerobic, catalase-negative Gram-positive cocci, that is non-motile and whose cells occur singly, in pairs, or in short chains. The organism only grows close to the hemolysis zone of S. aureus's colonies on blood agar. After sub-culturing, it produces pinpoint, non-hemolytic, non-pigmented colonies without $S$. aureus. In our study, after $48 \mathrm{~h}$ incubation (other publication claimed after $72 \mathrm{~h}$ incubation on blood agar [16]), this organism showed slight alpha-hemolytic activitiy (as shown in Fig. 2b). There was no difference in growth under $5 \% \mathrm{CO}_{2}$ or anaerobic conditions.

Biochemical methods are not reliable for $H$. ovis identification. Several studies have reported the misidentification of $H$. ovis as Granulicatella adiacens by various biochemical methods $[12,16,17]$. In this particular case, phenotypic characterization using the Vitek2 GP system gave an inconclusive result. However, the biochemical results with $H$. ovis in our study showed a marked disparity with the results of $H$. ovis CCUG 374411 and CCUG 39041 (as shown in Table 2, in which only different results are shown). To confirm this disparity, further experiments are needed. MALDI-TOF mass spectrometry was first used to identify $H$. ovis at the species level in our study. It presented a low log score value of 1.637 , but the matching strain result (H. ovis DSM $21504 \mathrm{~T}$ DSM) provided a valuable reference. This was due to the limited data in the MALDI-TOF database (Maldi-Biotyper database), which does not contain data on the various subspecies of $H$. ovis. In the future, the MALDI-TOF databases are expected to be expanded. 16S rRNA gene sequencing confirmed the species level identification in our study and we deposited the sequence in the GenBank database under accession number MG188744. Compared with the original sequence deposited by Collins and colleagues under Genbank accession number NR027228, our sequence differed by 15 base pairs. We also mapped phylogenetic tree based on data obtained from the GenBank database (1400 bp of sequence) using the maximum likelihood method (MEGA, version 6.0)(all original sequence data see Additional file 2). As shown in Fig. 4, H. ovis Tongii identified in our study was closely related to $H$. ovis, but differed from other $H$. ovis strains. According to the phenotype, genotype and clinical significance of our isolate, we consider it might to be a new subspecies of $H$. ovis. With the rapid development of whole genome sequencing, new genomic tools will help to minimize error and ensure accurate identification of bacterial species.

This article also reviewed previous literature about $H$. ovis infection published by several authors. The main clinical and microbiological characteristics of $H$. ovis infections are shown in Table 3. In 1999, this bacterium was first recovered from a mixed infection in sheep, but at this time the clinical significance of $H$. ovis was then unknown [2]. In 2003, Post and colleagues first described the isolation of $H$. ovis from cattle. Most anaerobes were assumed to grow polymicrobially. However, when in combination with Escherichia coli, H. ovis was associated with lesions in multiple tissues suggesting an etiological role in valvular endocarditis [18]. In 2004, relatively pure culture of $H$. ovis from both abscess and transtracheal wash samples indicated that this organism may pathogenic in the lungs of horses [16]. In 2008, 55 cases of bovine valvular endocarditis were collected and $H$. ovis $(18,33 \%)$ represented the second most common isolate recovered mainly as a pure culture [17]. This high level of prevalence demonstrated that $H$. ovis 


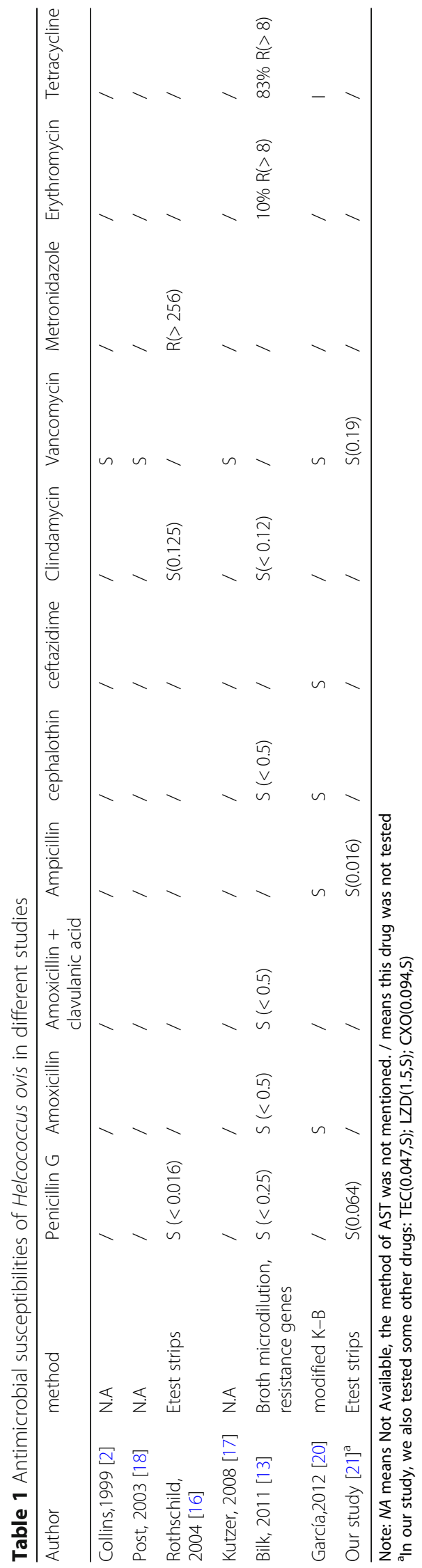


Table 2 Biochemical results for $\mathrm{H}$. ovis isolate compared to results obtained for $\mathrm{H}$. ovis control strains

\begin{tabular}{llll}
\hline Phenotypic characteristic & H. ovis control strains & H. ovis in \\
\cline { 2 - 3 } & CCUG 37441 & CCUG 39041 & our study \\
\hline$\beta$-Galactosidase & + & + & - \\
a-Glucosidase & + & + & - \\
Cyclodextrin & - & + & + \\
Leucine aminopeptidase & + & + & - \\
$\beta$-Glucuronidase & + & + & - \\
sorbitol & - & - & + \\
N-Acetyl-D-Glucosaminidase & - & - & + \\
Mannitol & - & - & + \\
Methyl- $\beta$-D-glucopyranoside & - & - & + \\
Pullulan & - & - & + \\
Raffinose & - & - & + \\
Trehalose & - & - & + \\
Arginine dihydrolase & - & - & + \\
\hline
\end{tabular}

Note: ${ }^{a}$ We didn't test $H$. ovis control strains, and the results of $H$. ovis control strains came from the results of Collins' and Kutzer's studies

was an emerging pathogen in bovine valvular endocarditis. In 2009, H. ovis was isolated from a sheep [19], where is was proposed to be the primary pathogen of pleuritis and bronchopneumonia. In 2012, García and coworkers reported that $H$. ovis was the dominant organism isolated from the lungs of a goat with pulmonary abscesses and purulent bronchopneumonia, again suggesting that $H$. ovis may play an etiologic role [20]. In the same year, Schwaiger proposed that the detection of $H$. ovis in four samples might

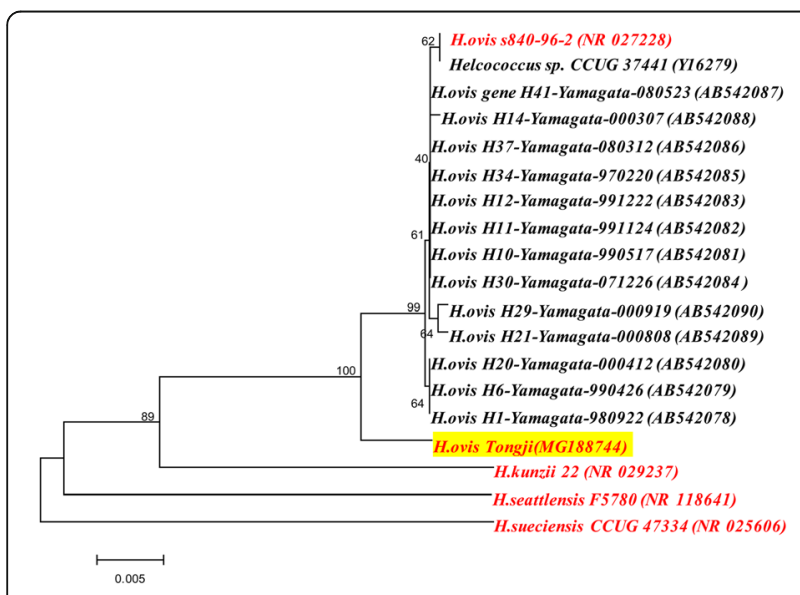

Fig. 4 Phylogenetic tree of Helcococcus ovis and other Helcococcus genus. Other compared Helcococcus genus include Helcococcus kunzii, Helcococcus sueciensis and Helcococcus seattlensis, and only type strains are focused. The length of $16 \mathrm{~S}$ rRNA gene sequence of Helcococcus pyogenica on the GenBank is only nearly 500 bp, which is not used for comparison. The lengths of all sequences included in the Phylogenetic tree are almost $1400 \mathrm{bp}$ indicate the involvement of this species in the pathogenesis of bovine mastitis [11]. In 2013, both $H$. ovis and $H$. kunzii were isolated from daily cows where they were potentially involved in uterine infections [12]. In 2014, H. ovis was isolated as a pure culture from the stomach contents of an aborted fetus [21]. This case was thought to be the first indication that H. ovis may cause bovine abortion. As shown in Table 3 , all isolates of $H$. ovis recovered to date were from animals i.e. sheep, bovine, horses and goats, where they caused infections such as valvular endocarditis, pulmonary abscess, pleuritis and bronchopneumonia, mastitis, and abortion. Furthermore, all isolates of $H$. ovis published to date had similar characteristics with virtually all being sensitive to vancomycin and sharing sequences similar (no more than 5 bp differences) to that of the type strain, H. ovis CCUG 37441.

To the best of our knowledge, we report here the first known human infection of $H$. ovis in a patient with an artificial eye. Although $H$. ovis was recovered along with $S$. aureus, dominant growth of $H$. ovis was detected in the artificial eye. In addition, considering the yellow-green appearance and the strong odor of the ocular prosthesis, anaerobic infection was more suspected. Therefore, we concluded that $H$. ovis might be the primary pathogen responsible for the eye infection. Interestingly, the patient reported no direct contact with animals, however, his work in a clothing factory brought him into contact with wool and cowhide for one month of the year. Three months had elapsed between the patient's last contact with wool and cowhide and the development of symptoms. Furthermore, neither his work colleagues nor relatives reported any similar infections. Although skin samples from the patient were also cultured, no significant growth was detected. The origin of this infection remains unclear and was not assessed by any microbiological data (i.e., we did not assess the patient's working and living environments). The patient did not report any recent drug use before admission, and according to physical examination and laboratory investigations, he was in relatively good health condition. Although he was a smoker and had a history of ocular surgery. Compared with the isolates of $H$. ovis previously reported in the literature, our isolate displayed distinct clinical and biochemical characteristics as well as genotypic differences (15 bp differences from the type strain). According to these data and the phylogenetic tree, we propose that our isolate might represents a new subspecies of $H$. ovis; however, further studies will be required to confirm this hypothesis.

We describe here the first known case of $H$. ovis in a young adult with an artificial eye infection. This case 


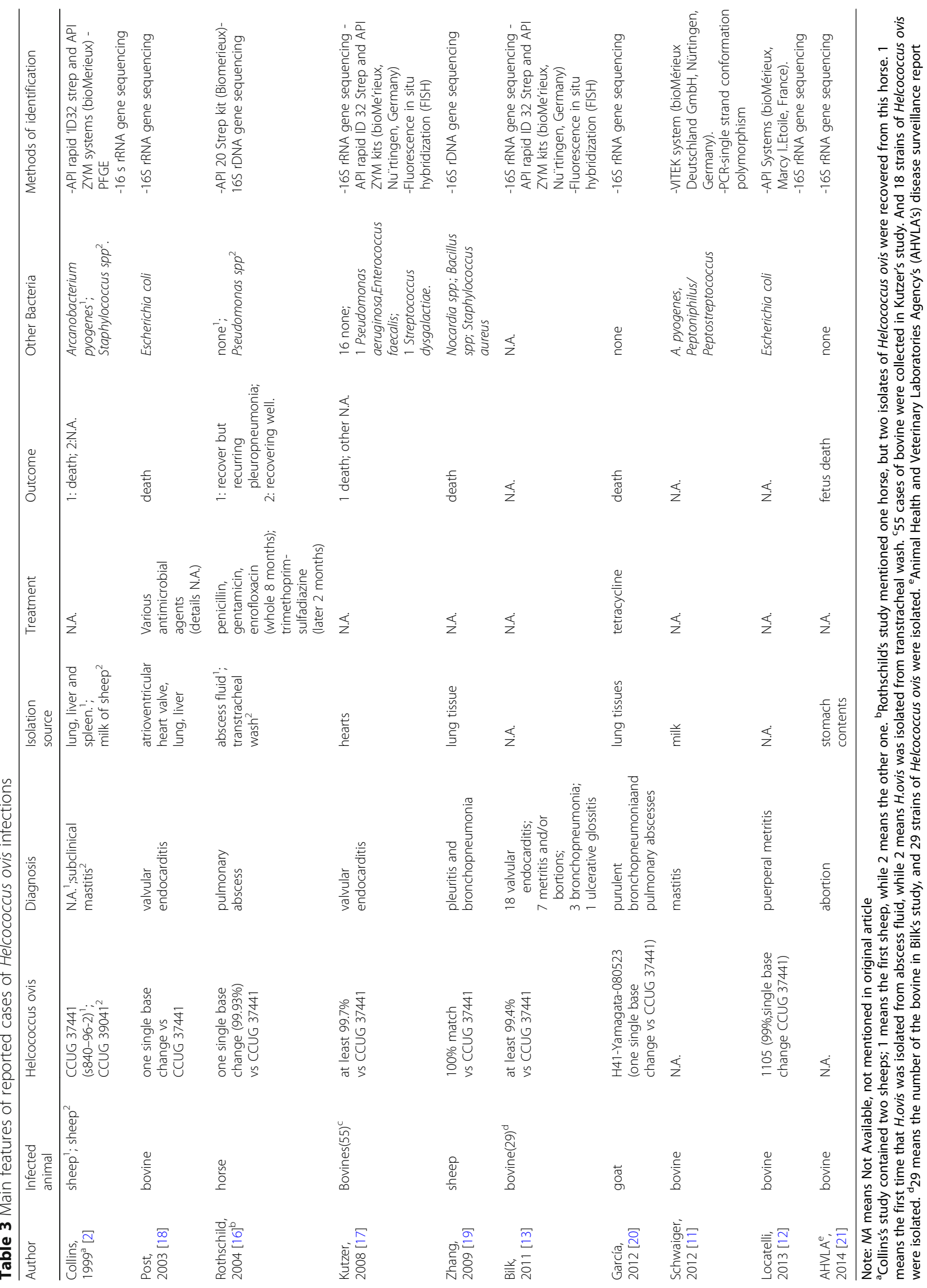


emphasizes the previously unreported invasive potential of this bacterial pathogen. Prospectively, the application of next generation sequencing tools would contribute to a more accurate classification of clinical strains.

\section{Additional files}

Additional file 1: Timeline. The timeline has covered this patient's relevant medical history, and the whole procedures during the period of the hospital. (DOC $42 \mathrm{~kb}$ )

Additional file 2: Sequence data. This file is divided into three parts. Part one is the $16 \mathrm{~S}$ rRNA gene sequence of $\mathrm{H}$. ovis in this article. Part two contains the sequences of type strains of Helcococcus kunzii, Helcococcus sueciensis and Helcococcus seattlensis. Part three contains all other sequences of $\mathrm{H}$. ovis that have been published in other articles. (DOC $80 \mathrm{~kb}$ )

\section{Abbreviations}

AST: Antimicrobial susceptibility testing; ; CLSI: Clinical and Laboratory Standards Institute; MALDI-TOF: Matrix-assisted laser desorption/ionization time of flight; Spn: Streptococcus pneumoniae

\section{Acknowledgements}

We thank Zhu Xuhui, Wang Yue, Tian Lei, Yan Shaozhen, Mao Lie, Luo Xuan, and Zhang Xuebing for careful reading of the manuscript and insightful comments. We thank Kate Fox, DPhil, from Liwen Bianji, Edanz Group China (http:// www.liwenbianji.cn/ac), for editing the English text of a draft of this manuscript.

\section{Funding}

This work was supported by The National Mega Project (2017ZX10103005-007). The funders had no role in study design, data collection and the decision to submit the work for publication.

\section{Availability of data and materials}

All data generated or analysed during this study are included within the article [and its additional files]. Sequence data of this organism that support the findings of this study have also been deposited in GenBank database with the accession number MG188744 (https://www.ncbi.nlm.nih.gov/ nuccore/MG188744)

\section{Authors' contributions}

All authors drafted and helped to write the manuscript. All authors read and approved the final manuscript. SZ wrote the manuscript and the response letter. ML contributed to the acquisition of clinical and microbiological data, and helped to write the manuscript. CZ contributed to the acquisition of clinical and microbiological data, and performed antimicrobial susceptibility testing on the clinical isolate. LY contributed to the acquisition of clinical and microbiological data. YJ participated in the sequence alignment of the $16 S$ sequences of the patient's strain. $Z Y$ contributed to the acquisition of clinical and microbiological data. LQ Helped to write the manuscript. LY contributed to the patient clinical follow-up. All authors have read and approved the manuscript, and ensure that this is the case.

\section{Ethics approval and consent to participate}

Not applicable, consent was obtained from the patient described in this report.

\section{Consent for publication}

Written informed consent was obtained from the patient for publication of this case report and any accompanying images. Written consent is available by request.

\section{Competing interests}

The authors declare that they have no competing financial interests.
Received: 1 December 2017 Accepted: 3 August 2018 Published online: 14 August 2018

\section{References}

1. Collins MD, Facklam RR, Rodrigues UM, Ruoff KL. Phylogenetic analysis of some Aerococcus-like organisms from clinical sources: description of Helcococcus kunzii gen. Nov., sp. nov. Int J Syst Bacteriol. 1993;43(3):425-9.

2. Collins MD, Falsen E, Foster G, Monasterio LR, Dominguez L, FernandezGarazabal JF. Helcococcus ovis sp. nov., a gram-positive organism from sheep. Int J Syst Bacteriol. 1999;49(Pt 4):1429-32.

3. Panackal AA, Houze YB, Prentice J, Leopold SS, Cookson BT, Liles WC, Limaye AP. Prosthetic joint infection due to "Helcococcus pyogenes" [corrected]. J Clin Microbiol. 2004;42(6):2872-4.

4. Collins MD, Falsen E, Brownlee K, Lawson PA. Helcococcus sueciensis sp. nov., isolated from a human wound. Int J Syst Evol Microbiol. 2004;54(Pt 5): 1557-60.

5. Chow SK, Clarridge JE 3rd. Identification and clinical significance of Helcococcus species, with description of Helcococcus seattlensis sp. nov. from a patient with urosepsis. J Clin Microbiol. 2014;52(3):854-8.

6. Martin I, Schwartzman J, Ruoff K. Multi-drug resistant helcococcus-like organism isolated from a chest abscess. Infectious Dis Rep. 2015;7(1):5754.

7. Vergne A, Guerin F, Lienhard R, Le Coustumier A, Daurel C, Isnard C, Marty N, Poyart C, Cattoir V. Identification and clinical significance of Helcococcus kunzii in human samples. J Clin Microbiol. 2015;53(8):2703-5.

8. Farid S, Miranda W, Maleszewski J, Sohail MR. Helcococcus kunzii prosthetic valve endocarditis secondary to lower extremity cellulitis. BMJ Case Rep. 2017; https://doi.org/10.1136/bcr-2017-219330.

9. Lotte R, Lotte L, Degand N, Gaudart A, Gabriel S, Ben H'dech M, Blois M, Rinaldi JP, Ruimy R. Infectious endocarditis caused by Helcococcus kunzii in a vascular patient: a case report and literature review. BMC Infect Dis. 2015;15:238.

10. Ngba Essebe C, Visvikis O, Fines-Guyon M, Vergne A, Cattoir V, Lecoustumier A, Lemichez E, Sotto A, Lavigne JP, Dunyach-Remy C. Decrease of Staphylococcus aureus virulence by Helcococcus kunzii in a Caenorhabditis elegans model. Front Cell Infect Microbiol. 2017;7:77.

11. Schwaiger K, Wimmer M, Huber-Schlenstedt R, Fehlings K, Holzel CS, Bauer J. Hot topic: bovine milk samples yielding negative or nonspecific results in bacterial culturing--the possible role of PCR-single strand conformation polymorphism in mastitis diagnosis. J Dairy Sci. 2012;95(1):98-101.

12. Locatelli C, Scaccabarozzi L, Pisoni G, Bronzo V, Casula A, Testa F, Allodi S, Pollera C, Toni F, Moroni P. Helcococcus kunzii and Helcococcus ovis isolated in dairy cows with puerperal metritis. J Gen Appl Microbiol. 2013;59(5):371-4.

13. Bilk S, Nordhoff M, Schulze C, Wieler LH, Kutzer P. Antimicrobial susceptibilities and occurrence of resistance genes in bovine Helcococcus ovis isolates. Vet Microbiol. 2011;149(3-4):488-91.

14. Vergne A, Guerin F, Lienhard R, Le Coustumier A, Daurel C, Isnard C, Marty N, Poyart C, Cattoir V. In vitro antimicrobial susceptibility of Helcococcus kunzii and molecular analysis of macrolide and tetracycline resistance. Eur J Clin Microbiol Infect Dis. 2015;34(10):2057-61.

15. Institute CaLS: M100-S25, performance standards for antimicrobial susceptibility testing; twenty-fifth informational supplement. 2015.

16. Rothschild CM, Oaks JL, Schaupp JK, Rurangirwa FR, Sellon DC, Hines MT. Helcococcus ovis isolated from a pulmonary abscess in a horse. J Clin Microbiol. 2004:42(5):2224-6.

17. Kutzer P, Schulze C, Engelhardt A, Wieler LH, Nordhoff M. Helcococcus ovis, an emerging pathogen in bovine valvular endocarditis. J Clin Microbiol. 2008:46(10):3291-5.

18. Post KW, Rushton SD, Billington SJ. Valvular endocarditis associated with Helcococcus ovis infection in a bovine. J Vet Diagn Invest. 2003;15(5):473-5.

19. Zhang Y, Cui J, Parkinson A, Hayes J, Ott K, Byrum B. Isolation of Helcococcus ovis from sheep with pleuritis and bronchopneumonia. J Vet Diagn Invest. 2009;21(1):164-6.

20. Garcia A, Risco D, Benitez JM, Martinez R, Garcia WL, Cuesta JM, Gomez L, Sanchez S. Helcococcus ovis isolated from a goat with purulent bronchopneumonia and pulmonary abscesses. J Vet Diagn Invest. 2012; 24(1):235-7.

21. AHVLA's disease surveillance report. Bovine abortions caused by Helcococcus ovis. Vet Rec. 2014;175(2):38-41.

\section{Publisher's Note}

Springer Nature remains neutral with regard to jurisdictional claims in published maps and institutional affiliations. 